Ann. Génét. Sél. anim., I970, 2 (2), I39-I44.

\title{
PROPORTIONS MENDÉLIENNES ANORMALES LIMITÉES A UN SEXE CHEZ LA POULE
}

\author{
P. MÉRAT \\ Station centrale de Génétique animale, \\ Centre national de Recherches zootechniques, 78-Jouy-en-Josas \\ Institut national de la Recherche agronomique
}

\section{RÉSUMÉ}

Une récapitulation des proportions mendéliennes a été faite dans notre population pour huit loci choisis pour la bonne correspondance qu'ils présentent entre phénotype et génotype. Pour trois d'entre eux $(R, W$, et $N a)$, le croisement ôt $A a \times$ 우우 a donne un rapport de ségrégation s'écartant de l'égalité de façon très hautement significative $(P<0,001)$ dans un sexe seulement (mâle pour les deux premiers, femelle pour le dernier). L'apparition simultanée de ces trois écarts par le hasard de l'échantillonnage est pratiquement exclue. L'interprétation paraît similaire pour les trois gènes. Une correspondance incomplète entre phénotype et génotype n'est pas compatible avec les résultats de tests de descendance et avec l'ensemble des faits concernant ces loci. Une sélection naturelle discriminatoire entre les classes zygotiques est très peu vraisemblable, car, en particulier, la proportion des sexes à l'intérieur de chaque phénotype montre un excès du type le plus nombreux, dans le sexe présentant une ségrégation anormale, par rapport à la catégorie correspondante de l'autre sexe. Il ne reste donc que l'hypothèse d'une fertilisation sélective.

Un second phénomène, concernant la méiose chez la femelle, est suggéré.

\section{INTRODUCTION}

Nous avons déjà fait état à plusieurs reprises de proportions mendéliennes anormales concernant des croisements où le mâle était hétérozygote, dans des populations de poules domestiques. Il s'agissait du gène $R$ de forme de la crête (MÉRAT, I962, I964 $a$ et $b$, I966 $a$, I969), des gènes $W$ de coloration de la peau (MÉRAT, rg66 b) et $C$ de coloration du plumage (MÉRAT, I966 c), enfin du gène $N a$ ou " cou nu " (MÉRAT, I970).

Un caractère commun à ces diverses anomalies mendéliennes est qu'elles sont limitées aux descendants d'un sexe. Ceci nous a conduit à récapituler brièvement ici les résultats correspondants, pour tenter d'en tirer une conclusion d'ensemble. 


\section{MATÉRIEL ET MÉTHODES}

Nous nous limiterons ici aux données fournies par un cheptel élevé à Jouy-en-Josas entre 1955 et 1969, dans lequel divers gènes sont maintenus en ségrégation à chaque génération.

Parmi ces gènes, nous en choisissons 8 pour lesquels les disjonctions comportent des effectifs suffisants, et dont l'identification des phénotypes est considérée comme sûre, la pénétrance de chaque allèle étant apparemment complète. Il s'agit des loci $C$ (présence /absence de coloration dans le plumage), I (mélanines noires ou non dans le plumage), $W$ (présence ou non de xanthophylles épidermiques), $R$ (crête en rose/simple), $B l$ (dilution/non dilution du noir du plumage), $P$ (crête en pois /simple), $C r$ (présence /absence d'une huppe), $N a$ (" cou nu "/plumage normal). Chacun de ces gènes comporte deux allèles connus dont l'un est dominant, l'hétérozygote étant bien distinct de l'homozygote récessif. Pour les descriptions correspondantes, nous nous référons à HutT (1949).

Les croisements analysés sont ceux où l'un des parents est hétérozygote et l'autre homozygote récessif, pour lesquels nous avons pu recueillir un volume de données suffisant.

L'identification du phénotype dominant ou récessif est faite à 1 jour pour les loci $C, R, P$, $\mathrm{Cr}, \mathrm{Na}$, à 8 semaines pour les autres, avec vérification des premiers. Le sexe est noté à 8 semaines d’âge et vérifié deux semaines plus tard, les risques d'erreur étant alors très faibles.

\section{RÉSULTATS}

Le tableau des nombres de descendants, classés par sexe et par phénotype,

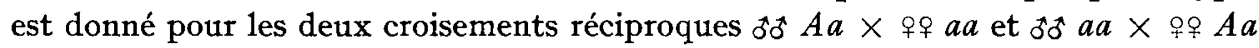
à chaque locus (tableau I).

Il apparaît clairement que les trois cas où l'on s'écarte de la proportion I/I de façon hautement significative $(P<0,00 I)$ concernent un seul sexe dans un croisement où le père est hétérozygote. Il s'agit des descendants mâles des croisements $\delta^{*} \sigma^{*} W w \times$ 우 $w w$ et $\delta^{*} \sigma^{*} R r \times$ 우 $r r$, des femelles dans le croisement ठํ $\mathrm{Na} n a \times$ 우 $n a n a$. Un quatrième écart aux valeurs théoriques, significatif seulement au seuil 5 p. Ioo, concerne les fils dans le croisement ơ $r r \times$ ofo $R r$.

\section{DISCUSSION}

Comme indiqué plus haut, nous avons signalé, dans des articles séparés, les faits relatifs aux gènes $W, R$ et $N a$. Nous nous intéresserons ici essentiellement à la comparaison entre eux des résultats obtenus pour divers gènes.

I. La réalité de chaque anomalie observée est très vraisemblable, au moins pour les trois auxquelles correspond un $\chi^{2}$ très élevé, et même en tenant compte de leur probabilité d'apparition aléatoire non plus isolée, mais parmi l'ensemble des résultats du tableau I. Ainsi, le plus petit de ces trois $\chi^{2}$, voisin de II, a une probabilité d'environ 0,0005 d'être dépassé dans un tirage aléatoire. Comme au total $32 \chi^{2}$ ont été calculés (8 loci, deux sexes, deux croisements réciproques), la probabilité de l'événement rare " apparition d'au moins un $\chi^{2}$ supérieur ou égal à II sur 32 tirages aléatoires " peut être estimée comme I - P $(o), P(o)$ étant la probabilité d'une valeur nulle dans une loi de Poisson de moyenne $m=0,0005 \times$ $32=0,0 I 6$. La quantité $\mathrm{I}-\mathrm{P}(0)$ est elle-même voisine de $0,0 \mathrm{I} 6$. En procédant de même, on trouve les probabilités relatives aux deux $\chi^{2}$ de valeur 15,7 et 20,9 voisines respectivement de 0,0012 et 0,00028 . 
TABLEAU I

Nombres de descendants classés par sexe et phénotype pour les croisements relatifs à chaque locus

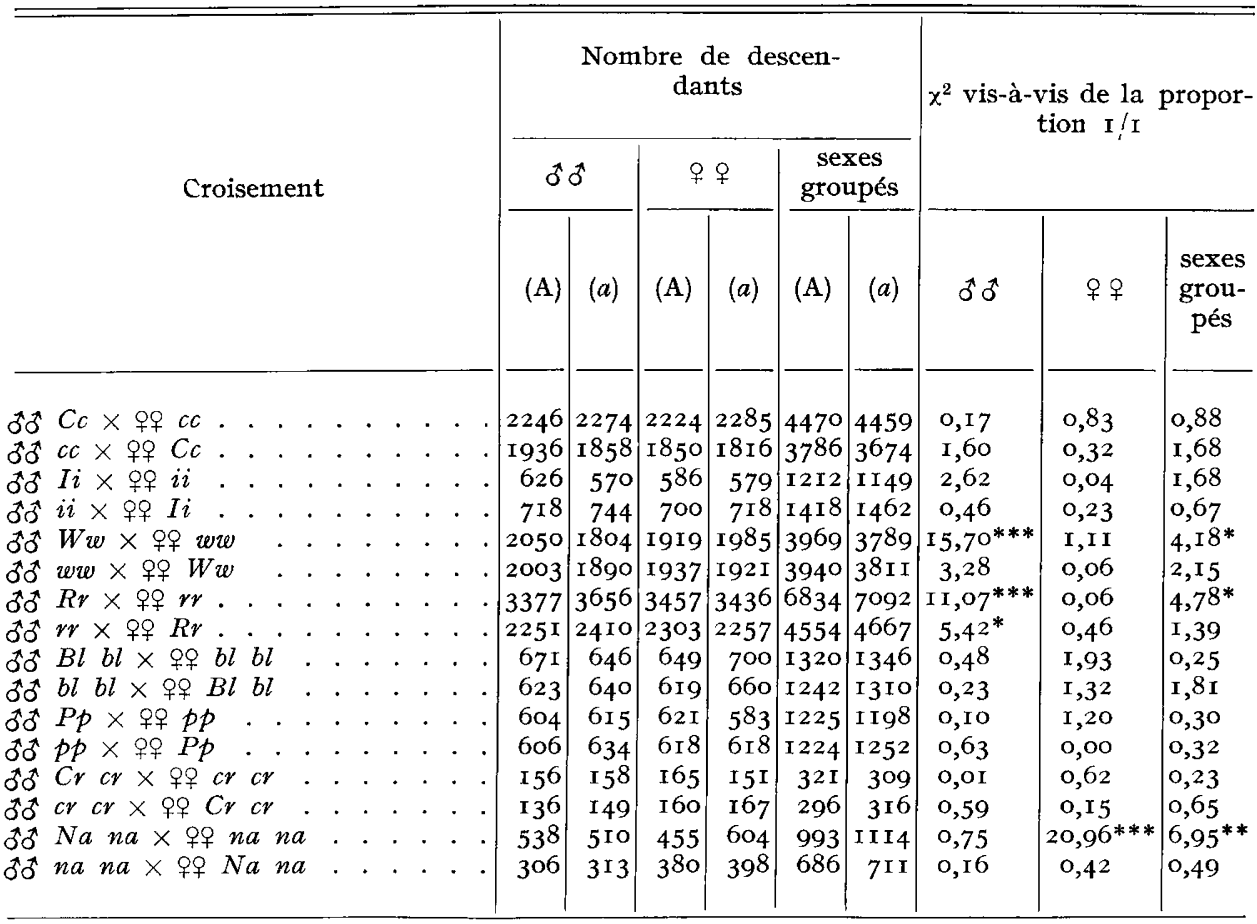

Remarques :

- (A) et $(a)$ représentent respectivement les phénotypes dominant et récessif.

.. $^{*}=$ significatif au seuil $5 \mathrm{p}$. I00; ${ }^{* *}=$ significatif au seuil $\mathrm{I}$ p. roo.; $* * *=$ significatif au seuil I p. Iooo.

Quant aux anomalies prises ensemble, on peut tenir raisonnablement pour impossible qu'elles soient toutes dues au hasard.

2. Chaque anomalie significative trouvée dans un sexe est bien limitée à ce sexe dans le croisement correspondant. Ceci ressort non seulement de ce que l'autre sexe se conforme de façon satisfaisante à la proportion théorique $I / x$, mais aussi du fait que le $\chi^{2}$ d'hétérogénéité entre sexes pour le rapport de ségrégation a une valeur significative. Cela est vrai des croisements ${ }^{\lambda} \sigma W w \times$ qo ww

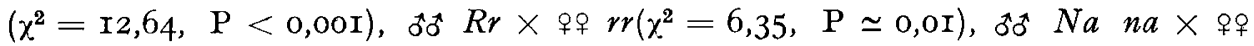
na $n a\left(\chi^{2}=\mathrm{I} 4,76, \mathrm{P}<0,00 \mathrm{I}\right)$, probablement aussi du croisement $\sigma^{\alpha} \alpha r \mathrm{r} \times$ 우 $R r$ $\left(\chi^{2}=4,50, \quad \mathrm{P}<0,05\right)$.

3. Nous avons signalé antérieurement (MéRAT, I962, I966 $b$, I969) que, dans chacun de ces cas, une correspondance non totale entre phénotype et génotype pouvait légitimement être exclue, non seulement parce qu'il s'agit de gènes à pénétrance réputée complète et d'anomalies de ségrégation limitées à un sexe, 
mais aussi de par les tests de descendance faits sur des individus appartenant à la catégorie la plus nombreuse.

Nous avons été amené à rejeter, dans ces articles, l'hypothèse d'une mortalité embryonnaire (ou post-embryonnaire pour le gène $W$ ) plus grande dans la catégorie trouvée la moins nombreuse. Les raisons en sont les mêmes dans les trois exemples où le père est hétérozygote. Tout d'abord, dans les croisements correspondants, le taux d'éclosion peut être considéré comme très " normal " et est au moins égal à la moyenne de la population entière pour les mêmes années. Mais l'argument qui nous paraît le plus déterminant, semblable également pour les trois loci, est la présence d'une proportion des sexes déviée non dans une seule catégorie phénotypique, comme on s'y attendrait si une élimination naturelle précoce frappait davantage un génotype dans un sexe, mais en sens contraire dans les deux catégories, alors que cette proportion des sexes, en réunissant les deux phénotypes, ne s'écarte pas sensiblement de I/I (tableau 2).

TABLEAU 2

Proporlion des sexes par catégorie phénotypique dans les trois croisements donnant un rapport de ségrégation nettement anormal

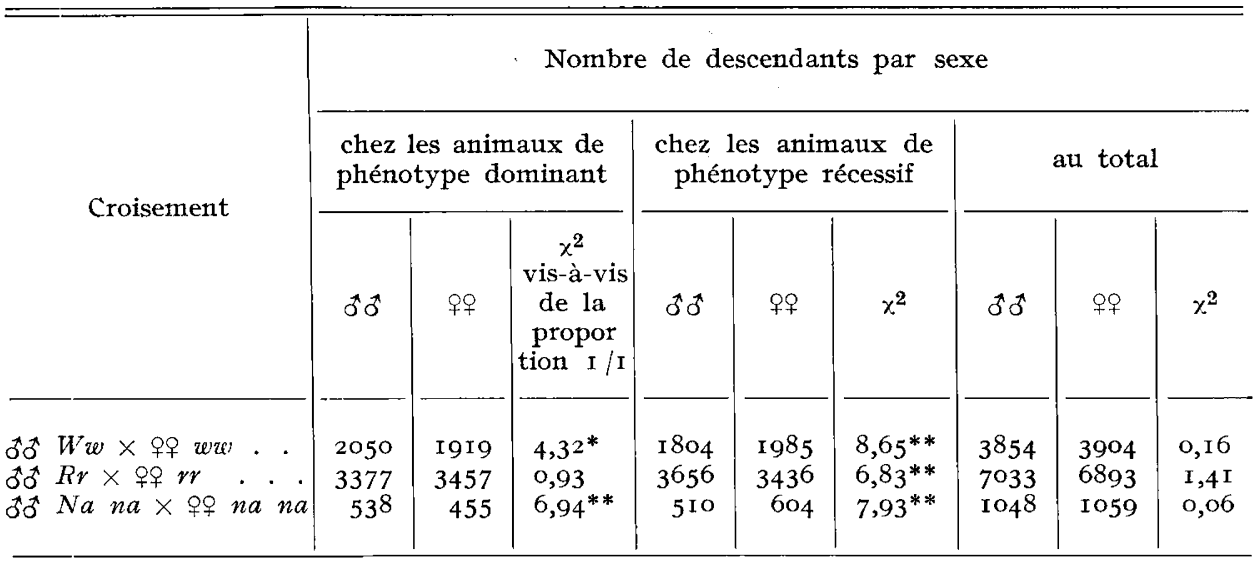

Remarque: $*=$ significatif au seuil 5 p. Ioo; $* *=$ significatif au seuil I p. roo.

Ainsi, il n'y a pas seulement un manque de la catégorie la moins nombreuse, mais un excès de la plus nombreuse par rapport à la classe correspondante dans l'autre sexe, et cet excès est significatif au seuil I p. Ioo dans les trois exemples. Cette répétition ne peut guère être un hasard.

Enfin, la comparaison avec le croisement réciproque apporte, au moins dans deux cas, un argument supplémentaire contre 1'hypothèse d'une sélection différentielle sur les zygotes. Au locus $N a$, on ne trouve aucun déficit décelable de " cou nu " chez les femelles dans le croisement đ̛ơ na na × 우 $N a n a$, et le $\chi^{2}$ d'hétérogénéité entre croisements réciproques, égal à 6,20 , est presque significatif au 
seuil I p. roo. Au locus $R$, quoiqu'il y ait dans le croisement ${ }^{*} \sigma^{*} r r \times q q R r$ un certain défaut de fils à crête en rose, ce dernier est plus accentué lorsque c'est le père qui est hétérozygote, et le $\chi^{2}$ d'hétérogénéité entre les deux croisements est égal à 3,5I $(\mathrm{P} \simeq 0,05)$. Au locus $W$, ce même $\chi^{2}$ n'est pas significatif (égal à 2,35$)$, mais, là aussi, l'écart à la proportion $\mathrm{I} / \mathrm{I}$ chez les mâles est plus net dans le croisement ơ $W w \times$ कㅇ $w w$.

4. Si la cause des trois ségrégations aberrantes évoquées ci-dessus ne réside ni dans une correspondance incomplète entre phénotype et génotype, ni dans une sélection zygotique, la seule hypothèse qui reste est celle d'une proportion anormale des gamètes à la fertilisation. Comme nous l'avons indiqué dans nos articles antérieurs pour chaque cas en particulier, ceci implique une interaction entre le gamète mâle, porteur de l'allèle dominant ou récessif, et l'ovule, possesseur de l'hétérochromosome $Z$ ou $W$, c'est-à-dire une fertilisation sélective. Ce phénomène ne semble pas si exceptionnel chez la Poule, puisqu'on l'observe associé à trois gènes " marqueurs " sur huit dans notre troupeau. Il faudrait d'ailleurs y ajouter un fait analogue pour le croisement ơ $R r \times$ कq $R r$ (MÉRAT, I969) ainsi que dans la descendance mâle de deux pères particuliers pour le croisement $\sigma^{*} C c \times$ \&o $c c$ : pour l'un, il $\mathrm{y}$ avait un excès hautement significatif $\left(\gamma^{2}>\mathrm{Io}\right)$ de fils à plumage blanc $c c$ (MÉRAT, I966 c); pour l'autre, c'était l'inverse (données non publiées); la ségrégation était normale chez les filles dans les deux cas $\left({ }^{1}\right)$.

Pour le croisement ở $R r \times$ 우 $r r$, nous avons détaillé (MÉ́RAT, I969) les différences entre plusieurs populations pour la proportion des crêtes chez les mâles et noté, d'autre part, que nous n'observions de disjonction anormale pour le gène $R$ que chez les fils colorés dans les familles où le père était hétérozygote $C c$. Ces variations selon la population ou le génotype résiduel rendent encore moins vraisemblable 1'hypothèse d'une sélection zygotique.

La dernière observation - proportion anormale limitée aux mâles porteurs de l'allèle $C$ lorsque le père est double hétérozygote aux loci $R$ et $C$ - nous a fait préciser notre hypothèse de fertilisation sélective, au moins dans ce cas précis, en y ajoutant celle d'une "interaction " ou " compétition " entre noyaux spermatiques ayant pénétré l'ovule. Ceci, précisément, est concevable du fait de l'existence de la polyspermie chez les oiseaux (Piko, I96I).

Une question annexe est posée, du fait que, dans deux des trois cas où le croisement ${ }^{\circ} \sigma^{A} A a \times$ 우 $a a$ donne une proportion anormale dans un sexe, le croisement réciproque semble présenter la même tendance, moins marquée il est vrai. Il s'agit des loci $W$ et $R$ (cf. tableau $\mathrm{I}$ ). Ceci n'enlève rien aux arguments concernant la réalité du phénomène trouvé lorsque le père est hétérozygote, mais suggérerait un second mécanisme, relatif à la méiose chez la femelle, avec une probabilité différente d'expulsion de deux allèles dans un globule polaire suivant le " sexe " de l'ovule. Comme la proportion globale des sexes est normale, ainsi que le rapport de ségrégation chez les femelles, ce mécanisme ne pourrait concerner que le passage de 1'un ou 1'autre allèle dans le second globule polaire. Ceci est imaginable si les

(1) Enfin, dans le croisement $\delta^{\star} P p \times \phi q p p$, une hétérogénéité significative entre plusieurs populations est trouvée pour le rapport de ségrégation chez les mâles, mais non chez les femelles (données non publiées). 
loci impliqués sont assez éloignés du centromère pour rester souvent hétérozygotes après la première division réductionnelle par suite d'enjambement (BATEMAN, I960). De toute façon, sur ce point, la signification de nos données est encore insuffisante, et de nouveaux résultats seraient nécessaires. S'ils confirmaient l'existence d'un second phénomène, ce dernier et le premier, relatif à la fertilisation, pourraient avoir en commun de correspondre à une " compétition " entre noyaux, l'un pour le maintien dans le cytoplasme ovulaire, l'autre pour la fécondation.

Rę̧u pour publication en février 1970.

\section{SUMMARY}

ABNORMAL MENDELIAN PROPORTIONS LIMITED TO ONE SEX IN THE FOWL

A survey of mendelian proportions was made in our population for eight loci choosen for the good correspondence they allow between phenotype and genotype. For three of them $(R$, $W$, and $N a)$, the cross ơ $\sigma^{*} A a \times q$ 우 $a a$ gives a segregation ratio very significantly $(\mathrm{P}<.001)$ deviating from equality in only one sex (males for the first two, females for the last ons). Simultaneous appearance by random sampling in three cases is practically excluded. The interpretation appears similar for the three genes. An incomplete phenotype-genotype correspondence is not compatible with progeny test results and with the overall evidence concerning these loci. Differential selection between zygotic classes is very unlikely, especially as sex-ratios within phenotypes show an excess of the more numerous type, in the sex with abnormal segregation, over the same category in the other sex. The remaining hypothesis is therefore that of selective fertilization.

A second phenomenon, concerning female meiosis, is suggested.

\section{RÉFÉRENCES BIBLIOGRAPHIQUES}

Bateman N., 1960. Selective fertilization at the T-locus of the mouse. Genet. Res., 1, 226-238. HUTT F.B., 1949. Genetics of the fowl. Mc Graw Hill, New York.

MÉrat P., I962. Ségrégation anormale pour les allèles "crête simple " et "crête en rose " chez la Poule. I. Croisement ơ $R r \times$ irr. Ann. Biol. anim. Bioch. Biophys., 2, rog-rr7.

MÉrat P., I963a. Ségrégations anormales pour les allèles "crête simple " et "crête en rose " chez la Poule. III. Croisement ô $R r \times q R r$. Ann. Biol. anim. Bioch. Biophys., 3, 125-13r.

Mérat P., r963 b. Ségrégations anormales pour les alèlles "crête simple " et "crête en rose " chez la Poule. IV. Discussion d'ensemble. Ann. Biol. anim. Bioch. Biophys., 3, 133-I4r.

Mérat P., r 966 a. Interaction entre deux loci pour une ségrégation anormale chez la Poule. Ann. Biol. anim. Bioch. Biophys., 4, 427-429.

MÉrat P., I966 b. Irrégularités des proportions mendéliennes au locus $W$ chez la Poule domestique. Ann. Biol. anim. Bioch. Biophys., 6, 249-254.

MÉrat P., Ig66 c. Ségrégation anormale au locus $C$ chez la Poule domestique. Ann. Biol. anim. Bioch. Biophys., 6, 423-425.

Mḱrat P., I969. Données complémentaires sur des anomalies de ségrégation au locus $R$ chez la Poule, lorsque le père est hétérozygote. Ann. Génét. Sél. anim., 1, 227-235.

Mérat P., Proportion mendélienne anormale pour le gène "cou nu " chez la Poule domestique. Ann. Génét. Sél. anim., 2.

Prko I., I96r. I,a polyspermie chez les animaux. Ann. Biol. anim. Bioch. Biophys., 1, 324-384. 